\title{
VALIDATION OF AN ANALYTICAL METHOD FOR DETERMINATION OF 8-ALPHA-HYDROXYMUTILIN
}

\author{
T. YANKOVSKA ${ }^{1}$, N. STOILOVA ${ }^{1}$, M. PEYCHEVA ${ }^{1}$, \\ S. IVANOVA-GOCHEVA ${ }^{2} \&$ A. NAZAROV ${ }^{2}$ \\ ${ }^{1}$ Central Laboratory of Veterinary Control and Ecology (CLVCE), \\ Sofia, Bulgaria; ${ }^{2}$ Biovet AD, Peshtera, Bulgaria
}

\section{Summary}

Yankovska, T., N. Stoilova, M. Peycheva, S. Ivanova-Gocheva \& A. Nazarov, 2015. Validation of an analytical method for determination of 8-alpha-hydroxymutilin. Bulg. J. Vet. Med., 18, No 2, 112-122.

The objectives of this study were to evaluate the concentration of 8 -alfa-hydroxymutilin $(8 \alpha-\mathrm{HM})$ as marker residue for tiamulin (pleuromutilins group), to verify and validate an analytical method for the quantitative determination of 8-alpha-hydroxymutilin in porcine tissues (muscle and liver). Quantification of $(8 \alpha-\mathrm{HM})$ was performed with liquid chromatography-tandem mass spectrometry (LCMS/MS). This method was used to determine the residue of $8 \alpha-\mathrm{HM}$ in porcine tissue after administration of Rodotet premix. In order to assess the residues in porcine tissues, an analytical method for 8alpha-hydroxy-mutilin was verificated and validated in line with accordance of Commission Decision 2002/657/EC and VICH GL49.

Key words: 8-alfa-hydroxymutilin, hydrolysis, marker residue, method validation, MRL, tiamulin

\section{INTRODUCTION}

Tiamulin is a pleuromutilin antibiotic licensed for use in veterinary medicine (Anonymous, 1999). One of the metabolites of tiamulin is 8-alpha-hydroxymutilin (8$\alpha-H M)$. Quantification of the analyte was performed with liquid chromatography-tandem mass spectrometry (LC-MS/ MS). Maximum residual levels (MRLs) of tiamulin have been established in Commission Regulation No 37/2010 (Anonymous, 2010) which lays down the presented marker residue for tiamulin as the sum of metabolites that may be hydrolysed to $8 \alpha-\mathrm{HM}$. The Regulation established MRLs of $100 \mu \mathrm{g} . \mathrm{kg}^{-1}$ for porcine muscle and $500 \mu \mathrm{g} \cdot \mathrm{kg}^{-1}$ for porcine liver. The available analytical method is further used for determination of $8 \alpha$-HM. It was apply for determination of drug residues in porcine tissues, which were produced as part of a residue study with Rodotet premix.

Validation procedure is performed following the requirements defined in Com- 
mission Decision 2002/657/EC (Anonymous, 2002) and Bioanalytical method validation. Linearity, within- and betweenrun accuracy and precision, limit of detection (LOD), limit of quantification (LOQ), specificity, applicability and practicability, carry-over on the LC-MS/MS instrument, susceptibility to interference were evaluated validation parameters are among routinely measured parameters.

The used verificated method contained a hydrolysis step (termed method A), similarly to the method developed by Department of Pharmacology, Toxicology and Biochemistry, Faculty of Veterinary Medicine, University of Gent (Anonymous, 2013). The method does not use internal standard norfloxacin $\mathrm{D}_{5}$, added from the beginning of analysis, because of the hydrolysis step which is not appropriate for this substance. The internal standard norfloxacin $\mathrm{D}_{5}$ was added before instrumental analysis to evaluate the matrix effect only. Additionally, to determine the behaviour of $8 \alpha-\mathrm{HM}$ (e.g. behaviour in matrix and freeze-thaw influence, the preparation of samples and stability of the analyte), an analytical method without hydrolysis step, described as method B, was also used.

A study was performed to evaluate the real content of $8 \alpha-\mathrm{HM}$ as marker residue for tiamulin and to verify the method for quantitative determination of $8 \alpha-\mathrm{HM}$ in porcine tissues (muscle and liver) developed and validated by the Department of Pharmacology, Toxicology and Biochemistry, Faculty of Veterinary Medicine, University of Gent for determination of $8 \alpha-\mathrm{HM}$ in rabbit tissues (Anonymous, 2013). The objectives of the study were on one hand, to determine the residue of marker compound of tiamulin in muscle and liver of pigs as part of a Biovet's residue study with Rodotet premix. On the other hand, the aim was to verify and validate an analytical method for the quantitative determination of 8-alphahydroxymutilin in porcine tissues performed in the routine practice of CLVCE as National Reference Laboratory.

\section{MATERIALS AND METHODS}

The study was performed under standardised laboratory conditions, standard operating procedures (SOPs) and special forms accompanying the SOPs for all experiments, given by Biovet. The analytical phase of the study was conducted according to the procedures described in the study plan and its amendment and observing the Principles of Good Laboratory Practice (Anonymous, 2009a).

\section{Chemicals and reagents}

Acetonitrile (ACN), etylacetate and methanol (Labscan, Dublin, Ireland) were of p.a. and HPLC grade. Formic acid, $\mathrm{HCl}$ and $\mathrm{NaOH}$ (p.a. grade) were supplied by Merck (Darmstadt, Germany). All working solutions were prepared with deionised water (18.2 M $2 . \mathrm{cm}$ resistivity) generated by ELGA system (Marlow, United Kingdom).

\section{Standards and standard solutions}

The 8-alpha-hydroxy-mutilin reference standard, stored in refrigerator, was purchase by Argus Chemicals srl (Chemical Reference Substance, Argus Chemicals srl, Italy). The assay of $8 \alpha-\mathrm{HM}$ resulted in more than $90 \%$ of 8 -alpha-hydroxymutilin (certificate of analysis provided by Argus Chemicals srl). Because the certificate of analysis doesn't give the exact amount of $8 \alpha-\mathrm{HM}$ it was decided to set the purity on $100 \%$ to create a worst case scenario. Argus Chemicals are the only provider of $8 \alpha-H M$ known to Biovet. 
Validation of an analytical method for determination of 8-alpha-hydroxymutilin

\section{Equipment}

Centrifuge Sigma 3K15 (Osterode am Harz, Germany); Nitrogen evaporation system, Block-Heater, Sartorius BP 221S analytical balance; Sartorius BP 601 technical balance; Polypropylene centrifuge tubes; Calibrated glassware for preparation of stock and working solutions of standards; Shaker; Autosampler vials with screw cap and teflon septum (Agilent Technologies, Germany); Solid-phase extraction manifold SUPELCO with Strata X cartridges $(200 \mathrm{mg}, 6 \mathrm{~mL})$, Phenomenex (USA); Inolab level $1 \mathrm{pH}$ meter WTW (Wissenschaftlich Technische Werkstätten, Weilheim, Germany) equipped with a combined glass electrode.

Instrumental analysis was provided with LC-MS/MS apparatus Surveyor LC Thermo Electron Corporation; TSQ ${ }^{\circledR}$ Quantum Discovery Max mass spectrometer, Thermo Electron Corporation (using the ESI positive mode); Vacuum pumps type EM30, Edwards; PC system, Dell with software Xcalibur ${ }^{\circledR}$, Thermo Electron Corporation. LC column Synergi Polar RP (50 x $2.6 \mathrm{~mm}$ i.d., $1.9 \mu \mathrm{m})$ with pre-column, Phenomenex.

\section{$L C-M S / M S$ conditions}

- Mobile Phase A - $\mathrm{H}_{2} \mathrm{O}: \mathrm{CH}_{3} \mathrm{OH}$ : $\mathrm{HCOOH}$ (90:10:0.1 /v:v:v), $0.01 \mathrm{M}$ Amm Formate;

- Mobile Phase B $-\mathrm{H}_{2} \mathrm{O}: \mathrm{CH}_{3} \mathrm{OH}$ : HCOOH (10:90:0.1/ v:v:v), 0.01M Amm Formate;

- The gradient eluting programme is presented in Table 1.

- Flow rate from $0.25 \mathrm{~mL} / \mathrm{min}$ and injection volume of $25 \mu \mathrm{L}$, column temperature $40{ }^{\circ} \mathrm{C}$;

- Scans, ion monitoring details, $\mathrm{m} / \mathrm{z}$ :

- 8-alpha-hydroxy-mutilin: $337.3 \rightarrow$ $283.3 ; 257.2 ; 337.3$

- Collision energy: $8 \mathrm{~V}$
Table 1. Gradient eluting programme

\begin{tabular}{llll}
\hline Time & $\mathrm{A} \%$ & $\mathrm{~B} \%$ & $\begin{array}{l}\text { Flow rate, } \\
\mu \mathrm{L} / \mathrm{min}\end{array}$ \\
\hline 0 & 90 & 10 & 250 \\
4 & 10 & 90 & 250 \\
7 & 10 & 90 & 250 \\
7.5 & 90 & 10 & 250 \\
10 & 90 & 10 & 250 \\
\hline
\end{tabular}

- Norfloxacin_D5 $325 \rightarrow 281$

- Collision energy: $14 \mathrm{~V}$

- Ionisation mode: Positive electrospray

\section{Samples}

The test item was stored at the test facility at $\leq-15^{\circ} \mathrm{C}$. The storage temperature was monitored continuously during the conduct of this validation study.

A sufficient amount (about $100 \mathrm{mg}$ ) of the test item 8-alpha-hydroxymutilin as reference substance together with a certificate of analysis arrived at the test facility. The test item was added to the test systems (i.e. blank porcine tissues) by spiking an appropriate amount of a solution. The calculations were done with respect to $8 \alpha-\mathrm{HM}$ since this is the marker residue of tiamulin. Homogenised blank porcine tissues were spiked with standard solutions and were used as quality control samples (QC sp). The QC spiked samples served as a control to check the acceptance of the extraction procedure and LCMS/MS run of evaluated unknown samples, especially for the hydrolysis step. The QC samples were prepared by spiking blank tissues at MRL levels of $8 \alpha-\mathrm{HM}$.

\section{Sample preparation procedures}

Method A (Anonymous, 2006): Sample extraction with hydrolysis (as described by the Department of Pharmacology, Toxicology and Biochemistry, Faculty of Veterinary Medicine, Gent). Place $1 \mathrm{~g}$ of minced tissue in a polypropylene centri- 
fuge tube. Add $5.0 \mathrm{~mL}$ of $0.01 \mathrm{M} \mathrm{HCl}$ in water/acetone $(50 / 50, \mathrm{v} / \mathrm{v})$. Shake the samples for $20 \mathrm{~min}$ and thereafter centrifuge them for $10 \mathrm{~min}$ at $4000 \mathrm{rpm}$. Transfer the upper liquid phase into a clean centrifuge tube and add $5.0 \mathrm{~mL}$ of hexane. Shake the samples for $10 \mathrm{~min}$ and centrifuge for $10 \mathrm{~min}$ at $4000 \mathrm{rpm} 0{ }^{\circ} \mathrm{C}$. Discarde the upper hexane phase and evaporate the remaining liquid phase under a stream of nitrogen at a temperature of $40 \pm 5{ }^{\circ} \mathrm{C}$ until ca. $2 \mathrm{~mL}$ remains in the tube. Add $3 \mathrm{~mL}$ of $7 \mathrm{M} \mathrm{NaOH}$ and vortex for ca. $15 \mathrm{~s}$. Spike the samples with an appropriate amount of 8-alpha-hydroxymutilin working standard solution. Place the samples in a water bath of $45{ }^{\circ} \mathrm{C}$ for $20 \mathrm{~min}$ to hydrolysis. Thereafter, add 2.0 $\mathrm{mL}$ of concentrated $\mathrm{HCl}$, vortex and cool down. Add $10.0 \mathrm{~mL}$ of ethyl acetate to the sample and shake the samples again for 20 min. Thereafter centrifuge the samples and transfer the upper ethyl acetate phase to a clean tube. Evaporate it under a gentle stream of nitrogen at temperature of $40 \pm 5{ }^{\circ} \mathrm{C}$ until dryness. Dissolve the residue in $250 \mu \mathrm{L}$ of initial LC mobile phase, add the appropriate volume of internal standard norfloxacin $\mathrm{D}_{5}$ and transfer into an autosampler vial. Inject an aliquot (25 $\mu \mathrm{L})$ in to the LC column.

Method B (Anonymous, 2009b): Place $2 \mathrm{~g}$ of tissue in a polypropylene centrifuge tube. Spike the samples at this point with an appropriate amount of 8-alpha-hydroxymutilin and norfloxacin $\mathrm{D}_{5}$ (internal standard) - working solutions. Add $10 \mathrm{~mL}$ McIlvine buffer with $0.1 \mathrm{M}$ Titriplex. Vortexing and thereafter centrifuge for $10 \mathrm{~min}$ at $4000 \mathrm{rpm}$ and $5{ }^{\circ} \mathrm{C}$. Filter the upper liquid phase into a flask and repeat the extraction. Rinse the filter with buffer and $0.1 \mathrm{M}$ Titriplex. Collect the supernatants. Solid-phase extraction: conditioning with methanol and water in series. Add the collected supernatants, rinse the cartridge with a mixture water/methanol $(95 / 5 \mathrm{v} / \mathrm{v})$ and dry for a $10 \mathrm{~min}$. Elute the analytes with $6 \mathrm{~mL}$ methanol. Evaporate to dry under stream of nitrogen. Dissolve the residue in $250 \mu \mathrm{L}$ of initial LC mobile phase, transfer the residue into an autosampler vial and inject an aliquot $(25 \mu \mathrm{L})$ onto the LC column.

The verificated analytical method was validated according Commission Decision 2002/657/EC (Anonymous, 2002). Linearity was done by a matrix matched calibration curve as linear regression plot of known concentration versus response of 5 $8 \alpha-\mathrm{HM}$ concentrations and evaluated using the correlation coefficient $r^{2}$. The term precision in this validation procedure covers the 'repeatability' or within-run precision and 'reproducibility' of between-run precision: The within-run conditions include the use of the same method on identical test material, in the same laboratory by the same operator using the same equipment within short intervals of time. The between-run conditions include the use of the same method on identical test material, in the same laboratory, but analysed on different days. The within-run and between-run precision were determined at four concentration levels per tissue. For each concentration level, analysis of 6 independently spiked samples was performed, 6 blank solvents samples and 18 blank tissues samples. It was expressed as the relative standard deviation (RSD, \%). Accuracy refers to the closeness of agreement between the true value of the analyte concentration and the mean result that is obtained by applying the experimental procedure a number of times $(n \geq 6)$. Accuracy calculated as (mean concentration found - spiked concentration)/spiked concentration $\times 100 \%$ was determined at three concentration 
levels per tissue. For each concentration level, analysis of 18 independently spiked samples were performed. In this study, the LOD was determined based on three calibration curves for 8-alpha-hydroxy-mutilin and was calculated using the intercept and slope of the curves according to following equation: $\mathrm{LOD}=3 \times$ Signal $/$ Noise . The limit of detection (LOD) (the smallest measured content from which it is possible to deduce the presence of the analyte with reasonable statistical certainty) was calculated using the intercept and slope of the curves according to following equation: $\mathrm{LOD}=3 \times$ Signal/Noise. The limit of quantification (LOQ) corresponds to the smallest measured content of an analyte above which the determination of the analyte can be made with a specified degree of accuracy and precision. Carry-over is the analyte concentration that possibly was determined in the solvent sample below the LOD level. The presence/absence of carry-over on the LC-MS/MS instrument was evaluated by injecting a blank solvent sample after the highest calibration sample.

The specificity refers to the ability of a method to distinguish between the analyte being measured and other substances. The specificity is predominantly a function of the measuring principle used. Since LC$\mathrm{MS} / \mathrm{MS}$ is used, the technique as such is already specific, since quantification is related to the molecular mass of 8-alphahydroxy-mutilin. Therefore, the specificity was demonstrated with respect to possible interferences of other endogenous compounds with the same retention time as 8alpha-hydroxymutilin. It was evaluated by the analysis of at least 18 blank tissue sample. Applicability refers to the commodities to which the method can be applied as described or with minor modifications. Practicability is a characteristic pro- cedure, dependent on the scope of the method. It is determined by requirements such as availability of standards, reagents and equipement, sample throughput, costs, safety. Robustness refers and relates to susceptibility to non-specific influences on the analytical results of certain experimental conditions, which can be subject to fluctuation $(\mathrm{pH}$, temperature, operator e.t.c.).

This parameter, validation of hydrolysis step and stability of the method were not evaluated according to standard protocol since sufficient control measures are taken at the test facility to minimize variations by strictly following the SOP . They were evaluated on the basis of information given by Department of Pharmacology, Toxicology and Biochemistry, Faculty of Veterinary Medicine, Gent (Anonymous, 2013).

\section{RESULTS}

The verificated analytical method was validated according to the widely accepted above mentioned criteria. Stability data described in literature are used and no experiments were done. The following parameters were demonstrated: for method A (with hydrolysis): linearity, withinrun and between-run accuracy, within-run and between-run precision, limit of detection (LOD), limit of quantification (LOQ), carry-over on the LC-MS/MS instrument, specificity, applicability and practicability, susceptibility to interference (Table 2).

The method without hydrolysis (method B) was evaluated for linearity, within-run and between-run accuracy and precision, limit of quantification (LOQ), limit of detection (LOD), specificity, applicability and practicability, susceptibi- 
Table 2. Validation parameters of the LCMS/MS method for $8 \alpha$-hydroxymutilin determination in porc tissue samples (spiked porcine muscle and liver)

\begin{tabular}{|c|c|c|}
\hline Matrix/sample parameters & $8 \alpha$-hydroxymutilin & Internal standard norfloxacin $\mathrm{D}_{5}$ \\
\hline \multicolumn{3}{|c|}{ Porcine muscle (spiked samples) } \\
\hline $\mathrm{MRL}, \mu \mathrm{g} / \mathrm{kg}$ & 100 & \multirow{9}{*}{$\begin{array}{l}\text { Corresponding concentration } \\
\qquad 100 \mu \mathrm{g} / \mathrm{kg}\end{array}$} \\
\hline Linear range, $\mu \mathrm{g} / \mathrm{kg}$ & $0-200$ & \\
\hline Recovery at MRL, $\%$ & 98 & \\
\hline $\mathrm{RSD}, \%(\mathrm{n}=54)$ & 26.53 & \\
\hline $\mathrm{LOD}, \mu \mathrm{g} / \mathrm{kg}$ & 23.39 & \\
\hline $\mathrm{LOQ}, \mu \mathrm{g} / \mathrm{kg}$ & 44.03 & \\
\hline Slope & -0.9721 & \\
\hline Intercept & 1.0167 & \\
\hline $\mathrm{R}^{2}$ & 0.9971 & \\
\hline \multicolumn{3}{|c|}{ Porcine liver (spiked samples) } \\
\hline MRL, $\mu \mathrm{g} / \mathrm{kg}$ & 500 & \multirow{9}{*}{$\begin{array}{l}\text { Corresponding concentration } \\
\qquad 100 \mu \mathrm{g} / \mathrm{kg}\end{array}$} \\
\hline Linear range, $\mu \mathrm{g} / \mathrm{kg}$ & $0-1000$ & \\
\hline Recovery at MRL, $\%$ & 99.12 & \\
\hline $\mathrm{RSD}, \%(\mathrm{n}=54)$ & 16.35 & \\
\hline $\mathrm{LOD}, \mu \mathrm{g} / \mathrm{kg}$ & 27.929 & \\
\hline $\mathrm{LOQ}, \mu \mathrm{g} / \mathrm{kg}$ & 46.837 & \\
\hline Slope & -3.5009 & \\
\hline Intercept & 1.0064 & \\
\hline $\mathrm{R}^{2}$ & 0.9952 & \\
\hline
\end{tabular}

lity to interference and carry-over on the LC-MS/MS instrument.

\section{Calibration curve linearity}

The calibration curves were prepared by analysing blank porcine tissue spiked with standard solutions at levels 25, 50, 100, $150,200,250,500,750,1000 \mu \mathrm{g} / \mathrm{kg} 8 \alpha-$ HM. The concentrations were calculated against the calibration curve, using internal standard correction by linear regression. The results are shown in Tables 2, 3 and 4 .

The within-run and between-run accuracy and precision were determined using blank porcine tissue spiked at 50, 100, 150 and $200 \mu \mathrm{g} / \mathrm{kg}$ of $8 \alpha-\mathrm{HM}(\mathrm{n}=9)$ for muscle and at 250, 500, 750 and 1000 $\mu \mathrm{g} / \mathrm{kg}$ of $8 \alpha-\mathrm{HM}(\mathrm{n}=9)$ for liver, i.e. the accuracy and precision (expressed as within-run and between-run precision) were calculated using spiked samples at the level of $1 / 2$ MRL, MRL, 1.5MRL and 2MRL. The accuracy fell within the range of $-20 \%$ to $+10 \%$. The LOQ was determined by analysing spiked samples $(\mathrm{n}=17)$ at a level of $50 \mu \mathrm{g} / \mathrm{kg} 8 \alpha-\mathrm{HM}$. The accuracy fell within the range of $-20 \%$ to $+10 \%$. The determined RSDs were also below the RSDmax value. Therefore, the LOQ was set at $50 \mu \mathrm{g} / \mathrm{kg}$ for all tissues examined.

The LOD data for both methods A and $\mathrm{B}$ are present in Tables 2 and 3.

For tissue analysis using LC-MS/MS, the carry-over from one sample to another was studied by injecting mobile phase and blank solvents-sample after the highest calibrator of 250 or $1000 \mu \mathrm{g} / \mathrm{kg}$. No peak was detected in the mobile phase samples 
demonstrating the absence of carry-over on the instrument for both methods $A$ and $\mathrm{B}$, because of acceptance for signal below of LOD.

The specificity was demonstrated with respect to possible interference of endogenous compounds with the same retention time as $8 \alpha-\mathrm{HM}$ and norfloxacin $\mathrm{D}_{5}$. It was evaluated by the analysis of in-house blank tissue samples. No peaks at the retention time of $8 \alpha-\mathrm{HM}$ and internal standard could be detected.

The method applied used standards, reagents and equipment that are commercially available. The method can be performed with a sample throughput that is normal for tissue analysis and at reasonable costs per sample. Only normal laboratory safety requirements have to be taken into account. The acceptance crite- ria for those parameters were followed.

Stability was demonstrated in solvent during storage: peak area of the test solution within $-10 \%$ of the area in the reference solution; in extract during analysis (QC), acceptance criteria: cfr. within-run accuracy \& precision; - in extract during storage (QC stab extr), acceptance criteria: cfr. within-run accuracy $\&$ precision; in matrix during storage (QCstab), acceptance criteria: cfr. within-run accuracy \& precision".

Chromatograms of standard solutions, blank tissues samples and spiked samples are given on Fig. 1.

\section{DISCUSSION}

In our experiments, the method was validating according to Commission Decision

Table 3. Results of the LOD calculation for $8 \alpha$-hydroxymutilin in porcine tissues for method A

\begin{tabular}{|c|c|c|c|c|}
\hline & \multicolumn{2}{|c|}{ Muscle } & \multicolumn{2}{|c|}{ Liver } \\
\hline & intercept & slope & intercept & slope \\
\hline \multirow{3}{*}{$\mathrm{R}^{2}$} & 0.0235305 & 0.00805228 & 0.00780564 & 0.00529145 \\
\hline & 0.9971 & & 0.9996 & \\
\hline & 0.0389911 & 0.00636149 & & \\
\hline $\mathrm{R}^{2}$ & 0.9900 & & & \\
\hline SD intercept & 0.0109 & 0.001196 & & \\
\hline Mean & 0.031264 & 0.007207 & & \\
\hline $\operatorname{LOD}(\mu \mathrm{g} / \mathrm{kg})$ & & & & \\
\hline
\end{tabular}

Table 4. Results of the LOD calculation for $8 \alpha$-hydroxymutilin in porcine tissues for method B

\begin{tabular}{lllll}
\hline & \multicolumn{3}{c}{ Muscle } & \multicolumn{2}{c}{ Liver } \\
\cline { 2 - 5 } & intercept & slope & intercept & slope \\
\hline $\mathrm{R}^{2}$ & 0.0113736 & 0.00181053 & 0.085 & 0.00225732 \\
$\mathrm{R}^{2}$ & 0.9902 & & 0.9901 & \\
Mean slope & 0.00995856 & 0.00203941 & 0.0293436 & 0.00285681 \\
\hline LOD $(\mu \mathrm{g} / \mathrm{kg})$ & & 0.00192497 & & 0.9925 \\
\hline
\end{tabular}




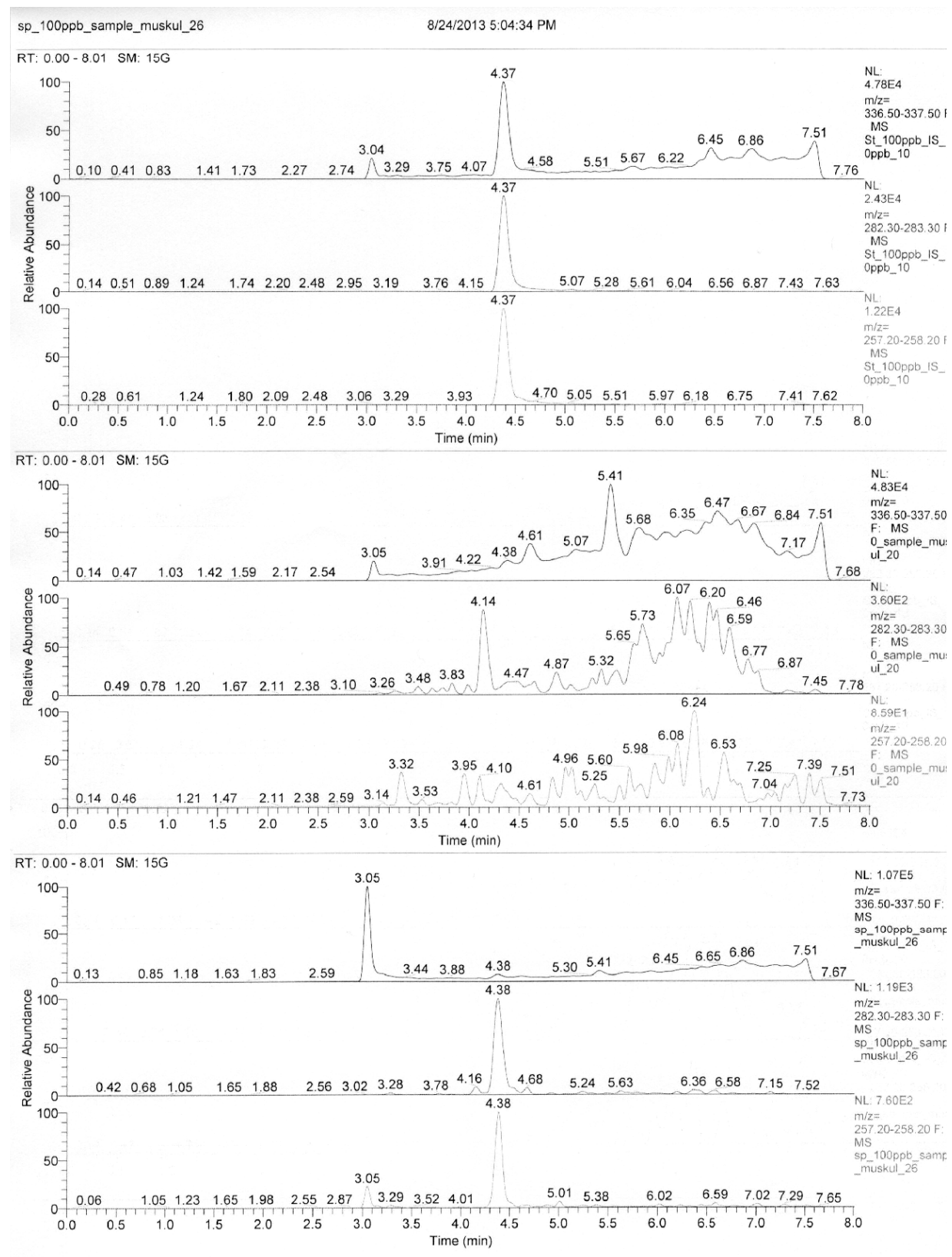

Fig. 1. LC-MS/MS chromatogram of standard at MRL level (porcine muscle), blank sample level (porcine muscle), spiked porcine muscle sample at MRL level. 
2002/657/EC (Anonymous, 2002). Several methods for determination of pleuromutilin residues in tissues with animal origin using different instrumental technices and sample preparation procedures have been published. Some of them are conducted by gas chromatography, HPLC and HPLC-MS (Markus \& Scherma, 1993a; Chen et al., 2006). The reported LOD of these methods ranged from $0.0014 \mathrm{ppm}$ for HPLC-MS (Schlusener et $a l ., 2003$ ) and 10 ppm for HPLC (Markus \& Scherma, 1993b). The sensitivity and the specificity by GC (Markus \& Scherma, 1993c) and traditional HPLC is lower than those of LC-MS/MS analysis. The methods for determination of tiamulin marker residue $8 \alpha$-HM are only few.

The described verification of method characteristics was done by evaluating the obtained data of experiments with porcine tissues only, as tiamulin produced by Biovet in Bulgarian veterinary practice is used only in swine. The described data were related only to the applicability of the method for porcine tissues and we do not have data on method's application (without or with adjustment of conditions) for other matrices. Experiments with other type matrixes (chicken muscle, liver, skin and fat) are forthcoming. Practically some of the steps in verification of an analytical method are represented without comparision of data and application of analytical procedures. Related to carry-over effect for verificated LC-MS/MS method, no peak was detected in the mobile phase samples demonstrating the absence of carry-over on the instrument for both methods A and B, because of acceptance for signal below of LOD. By the analysis of in-house blank tissue samples no peaks at the retention time of $8 \alpha-\mathrm{HM}$ and internal standard could be detected. The method is specific for the determination of 8-alpha-hydroxymutilin and norfloxacin $\mathrm{D}_{5}$ with respect to the interference of endogenous compounds for both methods A and $\mathrm{B}$, according to method, described by Gent team, and the data provided by Biovet from Huntigdon Life Science Ltd, England (Anonymous, 2006). The obtained data for applicability and practicability as verification method parameters were indentical to the method used in Gent with commercially available standards, reagents and equipment. Stability testing was not done as published data were used (Anonymous, 2006) and because of the opportunities given by Anonymous (2002).

Our method fulfilled the acceptance criteria, which, according to Knecht \& Stork (1974), required correlation coefficient $>0.99$.

As per VICH GL 49 (Anonymous, 2012) the precision for analyses carried out under repeatability conditions (withinrun): $\geq 10 \mu \mathrm{g} / \mathrm{kg}<100 \mu \mathrm{g} / \mathrm{kg}$ acceptable within-run precision (repeatability), $\% \mathrm{CV}$, is 15 and $\geq 100 \mu \mathrm{g} / \mathrm{kg}$ acceptable withinrun precision (repeatability), $\% \mathrm{CV}$, is 10 $\%$ ". The precision was also within the maximum RSD values, determined by Anonymous (2005) for analyses carried out under reproducibility conditions (between-run) $\mathrm{RSDmax}=2(1-0.5 \log C)$.

According to Anonymous (2002; 2005) the acceptance criteria for LOQ met the acceptance criteria, i.e. $\mathrm{LOQ}<\mathrm{MRL} / 2$; lowest point of the calibration curve; accuracy and precision - within the ranges specified.

The requirement for specificity (Heitzman, 1994; Anonymous, 2005) was also met: no endogenous substances with the same retention time as the analyte $(<$ LOD). Therefore, it was concluded that the method was specific for the determination of $8 \alpha-\mathrm{HM}$ and norfloxacin $\mathrm{D}_{5}$ with 
respect to the interference of endogenous compounds for both methods A and B. Criteria such as carry-over, applicability and practicability, were also fulfilled.

\section{REFERENCES}

Anonymous, 1999. EMEA/MRL/578/99-FINAL August 1999, NADA Number 140916.

Anonymous, 2002. Commission Decision 2002/657/EC implementing Council Directive 96/23/EC concerning the performances of analytical methods and the interpretation of results. Official Journal of the European Communities, L221, 8-36.

Anonymous, 2005. Notice to applicants, Veterinary medicinal products: Establishment of maximum residue limits (MRLs) for residues of veterinary products in foodstuffs of animal origin: Development and validation of a proposed regulatory method, EMEA/CVMP/573/00, vol. 8, 2005. http://ec.europa.eu/health/files/eudralex/vo 1-8/pdf/vol8_10-2005_en.pdf (Date last accessed 21 November 2014).

Anonymous, 2006. Research Laboratory Huntigton Life Science LTD England. Tiamulin and 8-Hydroxymutilin: Validation of methods of analysis for determining concentrations of tiamulin in porcine muscle and plasma and chicken egge and 8hydroxymutilin in porcine and rabbit liver and muscle and chicken liver, muscle and skin/fat, Final report 13.03.2006.

Anonymous, 2009a. Belgian GLP compliance monitoring programme manual, Scientific Institute of Public Health - Louis Pasteur, Brussels, 24/12/2009. https://www.wivisp.be/GLP/documents/BelgianGLPManua 1 ed3\%28revised2009\%29.pdf (Date last accessed 21 November 2014).

Anonymous, 2009b. BVL Group 5 Analysis „Test Method: Confirmatory Method for the determination of tetracyclines, quinolones, pleuromutilines, macrolides, lincosamides, sulfonamides, dapsone and trimethoprim in bovine and porcine muscle by LC-MS/MS", December 2009.

Anonymous, 2010. Commission Regulation (EU) No $37 / 2010$ on pharmacologically active substances and their classification regarding maximum residue limits in foodstuffs of animal origin. Official Journal of the European Union, L15, 1-72.

Anonymous, 2012. VICH GL49, Guidance for Industry, Studies to evaluate the metabolism and residue kinetics of veterinary drugs in food producing animals: validation of analytical methods used in residue depletion studies, 2012-Final. http://www. fda.gov/downloads/AnimalVeterinary/ GuidanceComplianceEnforcement/GuidanceforIndustry/UCM207942.pdf (Date last accessed 21 November 2014).

Anonymous, 2013. Development and validation of an analytical method for determination of 8-alpha-hydroxy-mutilin in rabbit tissues. Final report, Final version 08JAN13; Sponsor study number: RHuvepharma-2012-46; Test facility study number: 2012-FO-03; Department of Pharmacology, Toxicology and Biochemistry, Faculty of Veterinary Medicine (U Gent)

Chen, H.-C., S.-H. Cheng, Y.-H. Tsai \& D.-F. Hwang, 2006. Determination of tiamulin residue in pork and chicken by solid phase extraction and HPLC. Journal of Food and Drug Analysis, 14, 80-83.

Heitzman R. J., 1994, Veterinary Drug Residues. Residues in food producing animals and their products: Reference Materials and Methods, Report EUR 15127-EN, $2^{\text {nd }}$ edn, Blackwell Scientific Publications, UK, http://aei.pitt.edu/44343/1/A7247.pdf (24 November date last accessed).

Knech, J. \& G. Stork, 1974. Percentage and logarithmic procedures for calculation of calibration curves. Fresenius' Zeitschrift für analytische Chemie, 270, 97-99.

Markus, J. R. \& J. Sherma, 1993a. Liquid chromatographic determination of tiamulin hydrogen fumarate in feed premixes. Journal of AOAC International, 76, 444-446. 
Validation of an analytical method for determination of 8-alpha-hydroxymutilin

Markus, J. R. \& J. Sherma, 1993b. Liquid chromatographic determination of tiamulin hydrogen fumarate in complete swine meal feeds. Journal of AOAC International, $\mathbf{7 6}$, 449-450.

Markus, J. R. \& J. Sherma, 1993c. Gas chromatographic determination of tiamulin residues in swine liver. Journal of $A O A C$ International, 76, 451-452.

Schlusener, M. P., K. Bester \& M. Spiteller, 2003. Determination of antibiotics such as macrolides, ionophores and tiamulin in liquid manure by HPLC-MS/MS. Analytical and Bioanalytical Chemistry, 375, 942-947.
Paper received 10.06.2014; accepted for publication 12.10.2014

\section{Correspondence:}

Todorka Yankovska-Stefanova, $\mathrm{PhD}$

Central Laboratory of Veterinary Control and Ecology,

5 Iskarsko Shose str., 1528 Sofia, Bulgaria,

tel: +3592979 10 34, fax: +35929790742 e-mail: yankovska@clvce.eu 